\title{
Gestaltung von QM-Systemen in KMU unter Berücksichtigung von Aspekten grenzüberschreitender Kooperation
}

\author{
Nicole Petschick, Gerd Stegemann
}

\section{Problemstellung}

Die Volkswirtschaft der Bundesrepublik Deutschland ist durch eine starke internationale Verflechtung gekennzeichnet. Bei steigender Tendenz realisieren deutsche Unternehmen zirka ein Drittel ihrer Umsätze durch den Handel mit dem Ausland. Die Organisation eines florierenden Auslandsgeschäftes ist damit für viele deutsche Unternehmen Entwicklungsmotor und Zukunftssicherung zugleich. In |1| wird davon ausgegangen, dass dies in besonderem Maße auch für KMU zutrifft. Prognostiziert wird, dass sich der Anteil des Auslandsgeschäftes am Gesamtumsatz in mittelständischen Unternehmen bis 2005 annähernd verdoppeln wird. Auf Grund der EU-Erweiterung nach Mittel- und Osteuropa sind gerade fuir KMU der Region Berlin-Brandenburg erhebliche Impulse zu erwarten. Unter Berücksichtigung des als erwiesen geltenden Fakts, dass sich die Qualität von Produkten und Dienstleistungen national und international in zunehmendem Maße zu einem entscheidenden Wettbewerbselement entwickelt hat, müssen Aktivitäten zum Auslandsgeschäft (im Weiteren: grenzüberschreitende Kooperation) allerorts durch umfassendes betriebliches Qualitätsmanagement unterstützt, gefördert und abgesichert werden. Obwohl deutsche Produkte und Dienstleistungen traditionell im Ausland immer noch ein gutes Image haben, trifft diese Forderung in vollem Umfang auch und gerade für deutsche Unternehmen zu. Es ergibt sich ein
Gestaltungsbereich fuir das betriebliche Qualitätsmanagement mit zum Teil sehr differenzierten und speziellen Forderungen.

Nach Erkenntnissen in [2] findet dieser Gestaltungsbereich in den QM-Systemen von KMU trotz vorhandenen Regelungsbedarfs vielfach nur geringe Berücksichtigung. Es ist aus dem Grund zu empfehlen, notwendige Aktivitäten zum Qualitätsmanagement bezüglich grenzüberschreitender Kooperationen in vollem Umfang in das betriebliche QM-System zu integrieren. Dazu werden nachfolgend Vorschläge zur methodischen Vorgehensweise vorgestellt. Umfassende Untersuchungsergebnisse wurden gemeinsam mit Fachkollegen der EuroNorm Gesellschaft für Qualitätssicherung und Innovationsmanagement mbH in einem Leitfaden [2] publiziert.

\section{Prozessorientierung in der grenzüber- schreitenden Kooperation}

Voraussetzung der prozessorientierten Gestaltung qualitätsrelevanter Tätigkeiten zur grenziiberschreitenden Kooperation sind die Identifikation des Handlungsbedarfes und die auf dieser Grundlage zu treffende Entscheidung zur Realisierung qualitätsrelevanter Tätigkeiten im Sinne einer angemessenen Erfüllung der Forderungen und Erwartungen der interessierten Parteien des jeweiligen Unternehmens (s. Abb. 1).

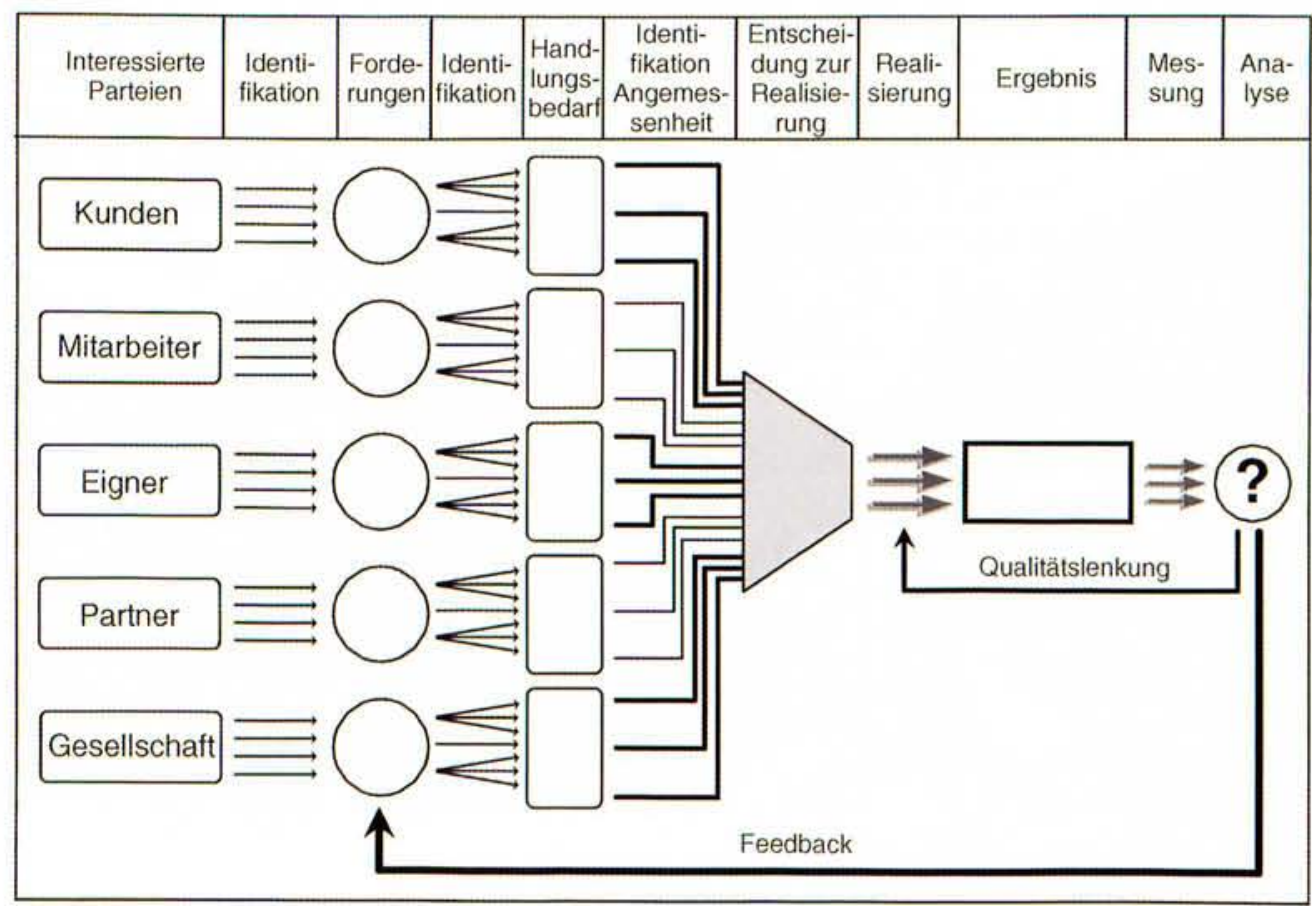

Abb. I: Ereigniskette zur Ableitung und Realisierung des Handlungsbedarfs interessierter Parteien 


\begin{tabular}{|l|l|l|}
\hline Kernprozesse & Managementprozesse & Unterstützungsprozesse \\
\hline K1 - Anbahnung von Außenhandelsbe- & M1 - Lenkung von Dokumenten und & U1 - Gestaltung des QMS \\
ziehungen & Aufzeichnungen & U2 - Qualitätsprüfungen \\
K2 - Marketing & M2 - Vertragsmanagement & U3 - Kommunikations- \\
K3 - Designtätigkeit, Entwicklung und & M3 - Vertragsprüfung & management \\
$\quad$ Konstruktion & M4 - Gestaltung Zahlungsverkehr & \\
K4 - Beschaffung und Beistellung & M5 - Personalmanagement \\
K5 - Verpackung & M6 - Außenhandelscontrolling & \\
K6 - Versand, Spedition, Logistik, Zoll- & M7 - Fehler-, Reklamations- und & \\
$\quad$ abwicklung & Beschwerdemanagement & \\
K7 - Ressourcenmanagement & M8 - Partnerschaftsmanagement & \\
K8 - Verwertung von Designergebnissen & M9 - Risikomanagement & \\
\hline
\end{tabular}

Tab. I: Identifizierte Geschäftsprozesse für die grenzüberschreitende Kooperation

Bei der Identifikation der Forderungen und Erwartungen der interessierten Parteien sind unbedingt die landesund unternehmensspezifischen und von inländischen Bedingungen abweichenden Prioritäten der ausländischen Kunden und Partner zu berücksichtigen. Landesspezifische Gesetze, Regelungen für Haftungsfragen, Vorschriften für den Marktzugang oder Versicherungsbedingungen sind Beispiele für abweichende und zu berüicksichtigende Forderungen der Gesellschaft im Partnerland. Eine besondere Herausforderung an die Managementtätigkeit stellt die Entscheidung zur angemessenen Beriicksichtigung relevanter Forderungen der interessierten Parteien dar. Als Ursache dafür ist der Umstand anzusehen, dass die Ziele der durch ein Unternehmen zu repräsentierenden Parteien gewöhnlich heterogen und teilweise gegenläufig sind, $d$. h. Zielkonflikte zwischen den interessierten Parteien nicht ausgeschlossen werden können und der Umfang realisierbarer Aktivitäten sehr begrenzt ist (Trichterprinzip in Abb. 1).

Eine prozessorientierte Gestaltung des betrieblichen QM-Systems vorausgesetzt, ist als erforderliche Basis qualitätsrelevanter Tätigkeiten des Unternehmens zur grenzüberschreitenden Kooperation zunächst die eindeutige Prozessidentifikation und -beschreibung anzusehen. Zu berïcksichtigen ist die Vernetzung spezifischer Prozesse zur Realisierung von Forderungen aus dem In- und Auslandsgeschäft mit denen mit Querschnittscharakter (s. Abb. 2).

Im Rahmen von Untersuchungen [2] wurden im Sinne der o. a. Forderung nach Integration notwendiger Aktivitäten des Qualitätsmanagements zur grenzüberschreitenden Kooperation in das betriebliche QM-System 8 Kern-, 9 Management- und 3 Unterstiitzungsprozesse (s. Tab. 1) identifiziert und diesen zu regelnde Aufgaben zugeordnet.

Für den Kermprozess 1 „Anbahnung von Außenhandelsbeziehungen" betrifft das z. B. Regelungen zur:

- zu vereinbarenden, verbindlichen Terminologie,

- Beschreibung von Ablauf, Gegenstand und Umfang der Außenhandelsrecherchen,

- Aufdeckung kultureller, historischer und politischer Aspekte der Zusammenarbeit,

- Beschreibung zu beriicksichtigender Aspekte des aufgabenbezogenen Außenhandelsrechts,

- Beobachtung von Ausschreibungen,

- Bestimmung der Art der Außenhandelsbeziehung sowie

- Erschließung möglicher Fördermöglichkeiten.

Im Mittelpunkt stehen die Prozesse mit wertschöpfender Wirkung (Kernprozesse) in der Beziehungskette Anforderungen und Zufriedenheit der Partner in der grenzüberschreitenden Kooperation (s. Abb. 3). Sie sind durch Management- und Unterstützungsprozesse zu flankieren und zu ihrer vollen Wirksamkeit zu führen. Abbildung 3 verdeutlicht, dass die Vernetzung der Kernprozesse im Sinne ihrer Prozessfolge in der Wertschöpfungskette erfolgt (dargestellte Prozessfolge ist als Beispiel zu werten). Die Vernetzungsrichtung der Management- und Unterstützungsprozesse ist vordergründig auf die Erhöhung der Wirksamkeit und Effizienz der Kernprozesse ausgelegt.
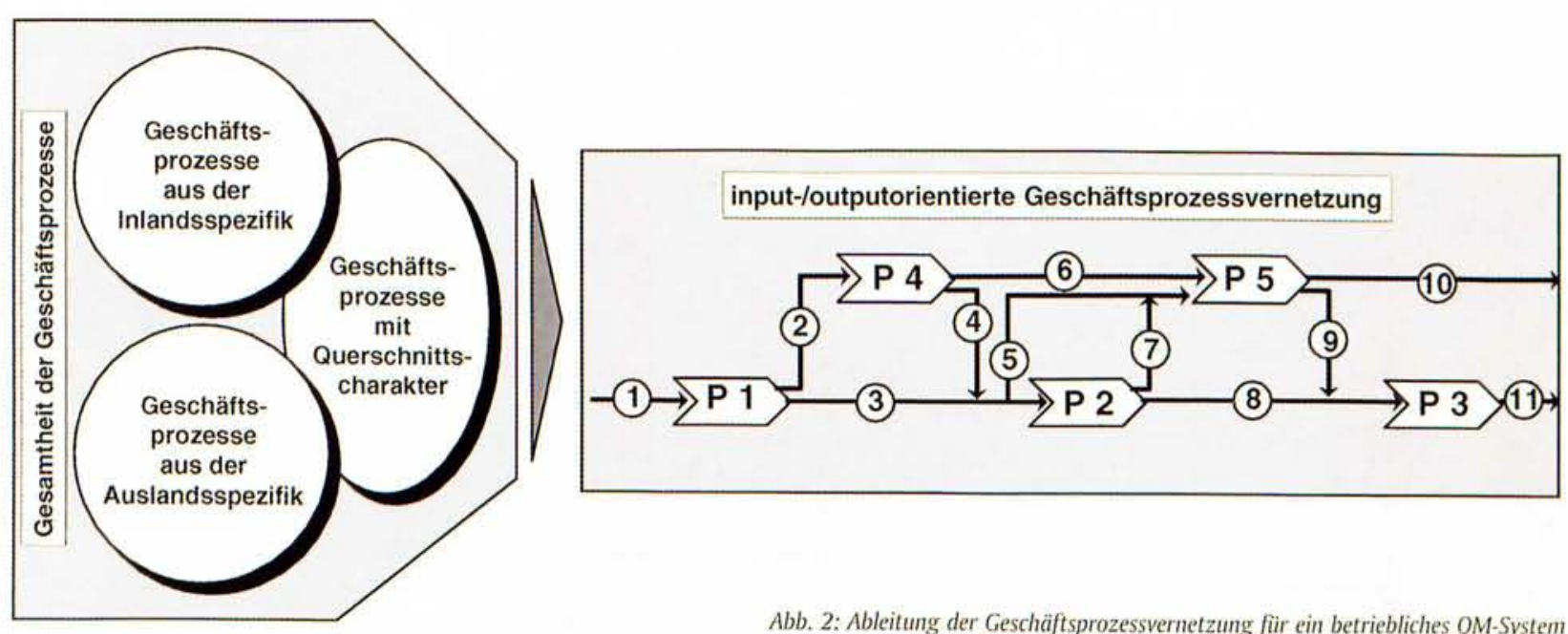

Abb. 2: Ableitung der Geschäftsprozessvernetzung für ein betriebliches QM-System 


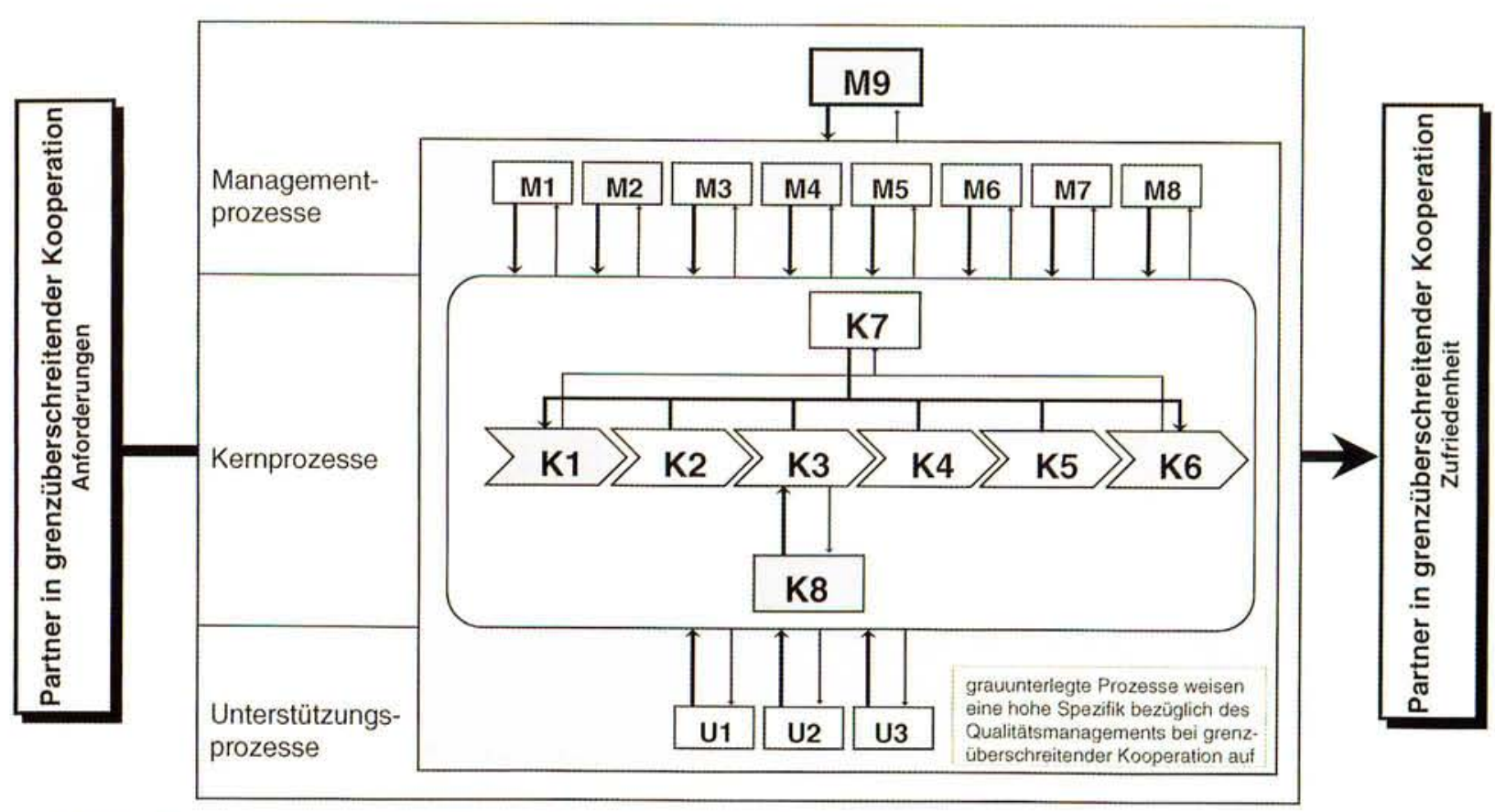

Abb. 3: Beispiel für die input-/outputorientierte Prozessvernetzung unter Aspekten der grenzüberschreitenden Kooperation

Eine wirksame Vernetzung dieser Prozesse setzt die unternehmensspezifische Kenntnis, Beschreibung und Realisierung ihrer In- und Outputs voraus. Abb. 4 und Tab. 2 zeigen am Beispiel ausgewählter Prozesse die Beschreibung der Input-/Outputbeziehungen.

Nach den Erkenntnissen der Autoren erfordert die Ermittlung und Beschreibung der Input-/Outputbeziehungen eine enge Zusammenarbeit der Prozesseigner, was:

- zu einer Präzisierung der Schnittstellenbeschreibungen fuihrt,

- zur Überwindung des Ressortdenkens im Sinne eines harmonisierten und alle Bereiche eines Unternehmens übergreifenden Bestrebens nach angemessener Erfüllung der Forderungen interessierter Parteien beiträgt und

- eine funktionsorientierte Unternehmensorganisation voraussetzt.

Es wird deutlich, dass wenige der identifizierten Prozesse (s. Tab. 1 und Abb. 3) nur ausschließlich auslandsspezifischen Regelungsbedarf zum Qualitätsmanagement haben (Prozesse mit hoher und sehr hoher Rege- lungsspezifik sind in Abbildung 3 besonders hervorgehoben). Daraus ergibt sich, dass Aspekte des Qualitätsmanagements zur grenzüberschreitenden Kooperation direkt in prozessorientierte QM-Systeme integrierbar sind. Notwendige Aktivitäten zum Qualitätsmanagement der grenzuiberschreitenden Kooperation sollten deshalb gewöhnlich in inhaltlich gleichlautende Prozesse des QM-Systems integriert werden.

Die im Einzelfall zu regelnden Prozesse werden wesentlich durch die Geschäftsfelder des Anwenders/Nutzers bestimmt. Einen Überblick zu möglichen Anwendern/ Nutzern des Know-hows zum Qualitätsmanagement für die grenzüberschreitende Kooperation vermittelt nachfolgende Übersicht:

- Unternehmen des produzierenden Gewerbes (öffentliche und private)

- Gewerbliche Dienstleistungsunternehmen

+ Groß- und Einzelhandel

+ Kreditinstitute, Versicherung

+ Verkehrsbetriebe

- Behörden

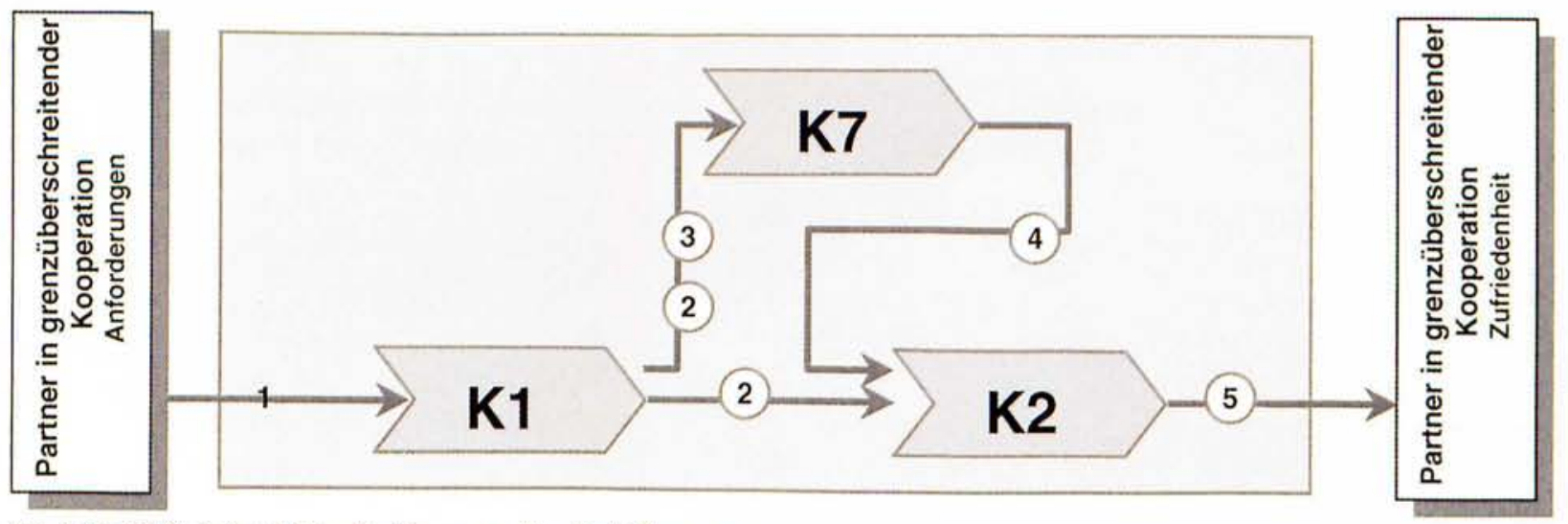

Abb. 4: Beispiel für die Input-/Outputbeziehung vernetzter Geschäftsprozesse 


\begin{tabular}{|c|c|c|c|c|}
\hline \multicolumn{2}{|r|}{ Input } & \multirow{2}{*}{$\begin{array}{c}\text { Prozess } \\
\text { K1 Anbahnung von Außen- } \\
\text { handelsbeziehungen }\end{array}$} & \multicolumn{2}{|r|}{ Output } \\
\hline 1 & $\begin{array}{l}\text { Wille/Bestreben zur Erweiterung } \\
\text { der unternehmerischen Tätigkeit } \\
\text { um das Auslandsgeschäft }\end{array}$ & & 2 & $\begin{array}{l}\text { - Marktsegmente, } \\
\text { Entwicklungstrends nach } \\
\text { Regionen/Ländern } \\
\text { - zu berücksichtigende regionale } \\
\text { und überregionale kulturelle, } \\
\text { historische und politische } \\
\text { Aspekte } \\
\text { - Varianten möglicher } \\
\text { Außenhandelsbeziehungen nach } \\
\text { Regionen und Ländern } \\
\text { - Finanzierungsvarianten } \\
\text { Forderungen an Art und Umfang } \\
\text { erforderlicher personeller, } \\
\text { materieller und finanzieller } \\
\text { Ressourcen }\end{array}$ \\
\hline $\begin{array}{l}2 \\
3\end{array}$ & $\begin{array}{l}\text { Finanzierungsvarianten } \\
\text { Forderungen an Art und Umfang } \\
\text { erforderlicher personeller, } \\
\text { materieller und finanzieller } \\
\text { Ressourcen }\end{array}$ & K7 Ressourcenmanagement & 4 & $\begin{array}{l}\text { - quantifizierter Ressourcenbedarf } \\
\text { - Plan zur } \\
\text { Ressourcenbereitstellung } \\
\text { - Maßnahmeprogramm zur } \\
\text { Aktivierung der Ressourcen } \\
\end{array}$ \\
\hline 2 & $\begin{array}{l}\text { - Marktsegmente, } \\
\text { Entwicklungstrends nach } \\
\text { Regionen/Ländern } \\
\text { - zu berücksichtigende regionale } \\
\text { und überregionale kulturelle, } \\
\text { historische und politische } \\
\text { Aspekte } \\
\text { - Varianten möglicher } \\
\text { Außenhandelsbeziehungen } \\
\text { nach Regionen und Ländern } \\
\text { - quantifizierter } \\
\text { Ressourcenbedarf } \\
\text { - Plan zur } \\
\text { Ressourcenbereitstellung } \\
\text { - Maßnahmeprogramm zur } \\
\text { Aktivierung der Ressourcen }\end{array}$ & K2 Marketing & 5 & $\begin{array}{l}\text { - Plan der Marketingaktivitäten, } \\
\text { wie Beteiligung an Messen oder } \\
\text { Bietergemeinschaften, Werbung, } \\
\text { Gewinnung von Partnern in } \\
\text { Zielregion/-land } \\
\text { - Entwicklung von } \\
\text { Marketinginstrumenten } \\
\text { - Zuarbeit zur strategischen } \\
\text { Geschäftsplanung } \\
\text { - Maßnahmeprogramm zur } \\
\text { Realisierung der } \\
\text { Marketingaktivitäten }\end{array}$ \\
\hline
\end{tabular}

Tab. 2: Input-/Outputbeschreibung zur Abb. 4

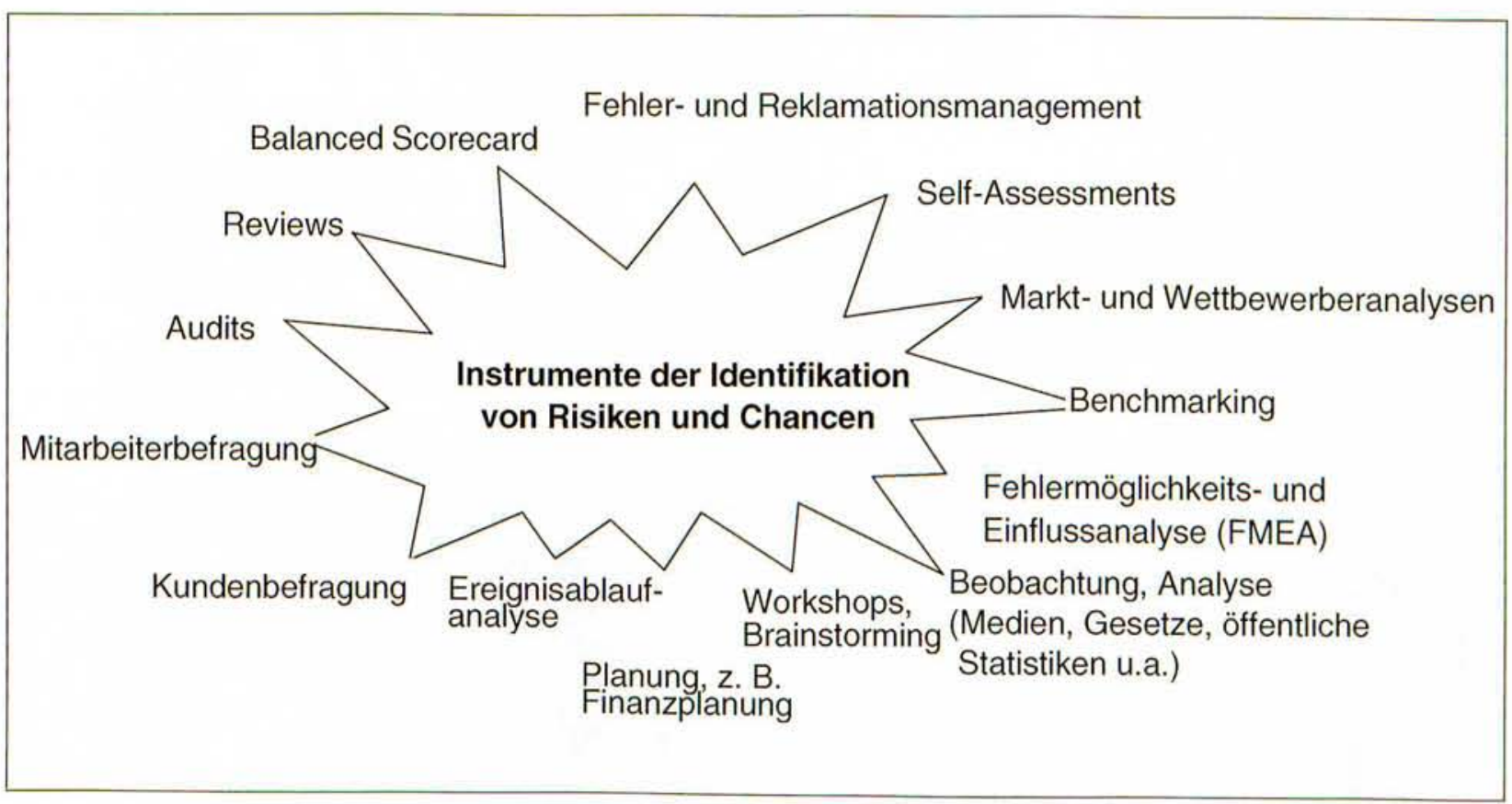




\begin{tabular}{|c|c|}
\hline Kategorie & Beispiele/Ursachen \\
\hline \multirow{7}{*}{ 1. Finanzielle Risiken } & - Währungsrisiken, Devisenbeschränkungen \\
\hline & - Forderungsausfälle \\
\hline & $\begin{array}{l}\text { - unzureichende Absicherung der Mindest-Finanzausstattung / zu } \\
\text { späte Finanzplanung }\end{array}$ \\
\hline & - fehlende Schärfe in Unternehmensstrategie \\
\hline & - Unkenntnis Ausschreibungen \\
\hline & - falsche/ fehlende Exportversicherung \\
\hline & - $\quad$ Liquiditätsrisiken, Inkasso \\
\hline \multirow{3}{*}{ 2. Prozessrisiken } & - ungeeignete Partner \\
\hline & - Vertrieb und Logistik nicht ausgereift \\
\hline & - Technologierisiken (Ausfall) \\
\hline \multirow{6}{*}{$\begin{array}{l}\text { 3. Produkt- und } \\
\text { Dienstleistungsrisiken }\end{array}$} & - Entwicklungsrisiken \\
\hline & - $\quad$ falsche Bewertung Marktvolumen \\
\hline & - $\quad$ unzureichende Produkt- / Dienstleistungsanpassung an den Markt \\
\hline & $\begin{array}{llll}\text { - } & \text { unzureichende Marktkenntnisse / Vorbereitung des } \\
\text { Auslandsgeschäftes } & & \\
\end{array}$ \\
\hline & $\begin{array}{llllll}\text { - } \quad \begin{array}{l}\text { unausgewogenes Angebot, } \\
\text { unzureichende Schulung }\end{array} & \text { B. fehlender Service oder } \\
\end{array}$ \\
\hline & - ungeeigneter Vertriebsweg \\
\hline \multirow{2}{*}{ 4. Sicherheits- und Sachrisiken } & - $\quad$ IT-Sicherheit \\
\hline & - Technologiesprünge \\
\hline \multirow{4}{*}{ 5. Rechtsrisiken } & - Vertragsrisiken \\
\hline & - $\quad$ unzureichende Beachtung landesspezifischer Vorschriften \\
\hline & - Missachtung Handelsbeschränkungen / Embargos / Sanktionen \\
\hline & - Verletzung Zoll- und Einfuhrbestimmungen \\
\hline \multirow{2}{*}{ 6. Beschaffungsrisiken } & - Beschaffungsengpässe \\
\hline & - Unterbrechung der Verkehrslogistik (Streik) \\
\hline \multirow{5}{*}{ 7. Absatzrisiken } & - $\quad$ regionales/landesspezifisches Konsumtionsklima \\
\hline & - $\quad$ Missachtung landesspezifischen Konsumtionsverhaltens \\
\hline & - unzureichender After-Sales-Service \\
\hline & - Weltmarktsituation \\
\hline & - globale Konjunkturentwicklung \\
\hline \multirow{3}{*}{ 8. Personenrisiken } & - $\quad$ unzureichende Vorbereitung der Mitarbeiter auf die neuen Märkte \\
\hline & - Fluktuation \\
\hline & - $\quad$ individuelle Fehler von Mitarbeitern \\
\hline \multirow{2}{*}{ 9. Umweltrisiken } & - Missachtung von Vorschriften/Gesetzen \\
\hline & - Naturgefahren \\
\hline \multirow{2}{*}{$\begin{array}{l}\text { 10. Politische /gesellschaft- } \\
\text { liche Risiken }\end{array}$} & - Imageverlust \\
\hline & - $\quad$ Ein- und Ausfuhr-Beschränkungen \\
\hline
\end{tabular}

Tab. 3: Ausgewälıte Risikokategorien der grenzüberschreitenden Kooperation

- Kammern

+ berufsständische Vertretung auf gesetzlicher Grundlage

+ kollegialer Spruchkörper eines Gerichts

- Verbände

Generell wird der Gestaltung des Risikomanagements im Rahmen der grenzüberschreitenden Kooperation eine große und iibergreifende Funktion zugeordnet, da sich die Risiken im Auslandsgeschäft teilweise erheblich von denen des Inlandes unterscheiden (s. Abb. 3).

Für die Identifikation von Risiken stehen verschiedene Instrumentarien zur Verfügung (s. Abb. 5).

Qualitätsaudits und -reviews, Balanced Scorecards, Fehlermöglichkeits- und Einflussanalysen, Self-Assessments, das Fehler- und Reklamationsmanagement sowie Ereig- nisablaufanalysen gehören zu den bewährten Methoden und Techniken des Qualitätsmanagements, welche auch für Aufgabenstellungen zum Risikomanagement nutzbar sind.

Es muss von einem erheblichen Spektrum verschiedener Risiken in der grenziiberschreitenden Kooperation ausgegangen werden (s. Tab. 3).

Letztlich sind im Rahmen der Prozessgestaltung Risikobewältigungsstrategien zu identifizieren und im Sinne integrierter Risikobetrachtung zu regeln (s. Abb. 6). Daraus folgt, dass Maßnahmen der Risikovermeidung, -minderung und -verlagerung strategisch iibergreifend als Geschäftsprozess zu regeln und gleichzeitig detaillierte Bewältigungsmaßnahmen in die betreffenden Geschäftsprozesse zu integrieren sind. 


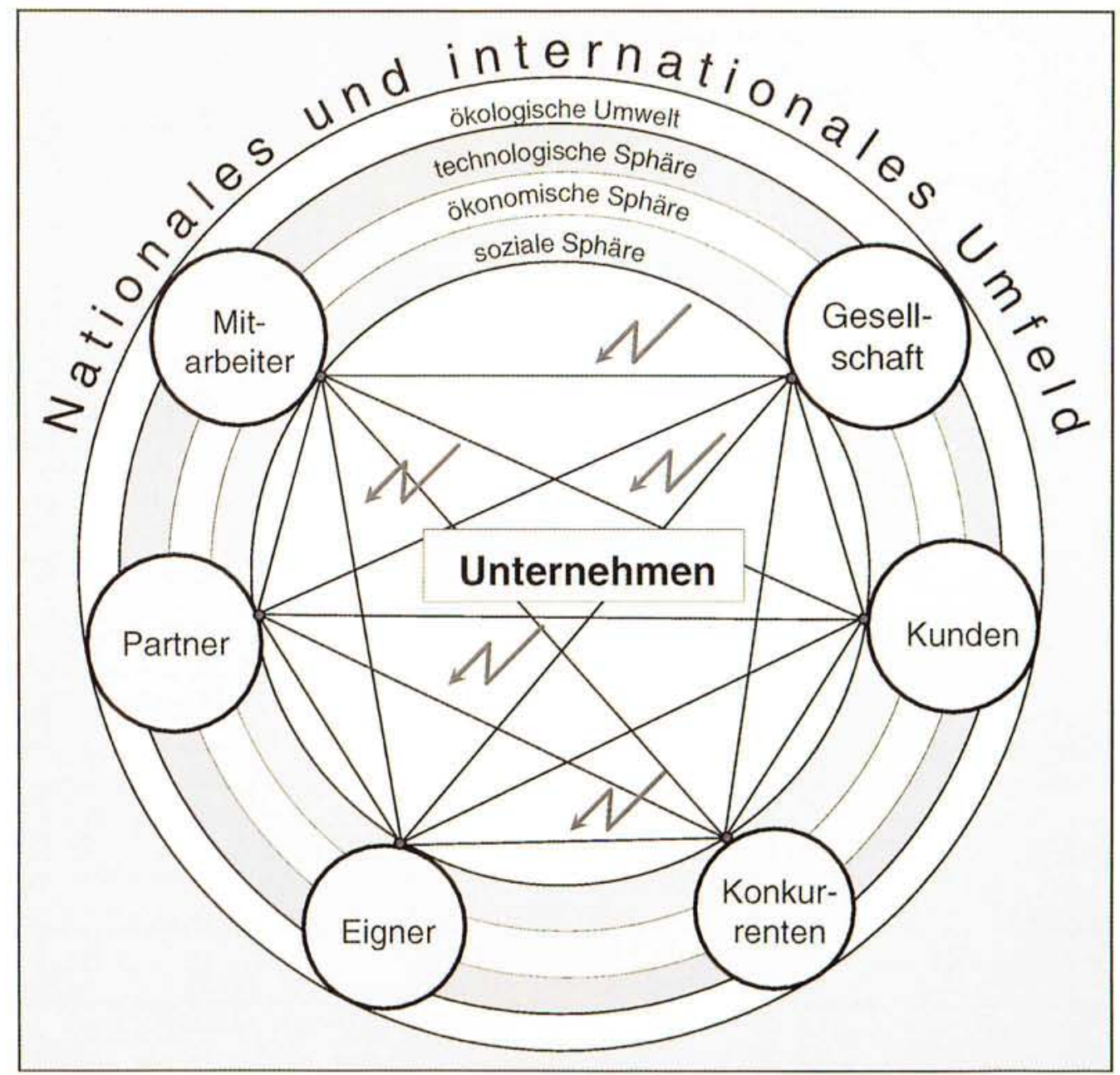

Abb. 6: Integrierte Risikobetrachtung in Unternehmen /verändert nach 3 |

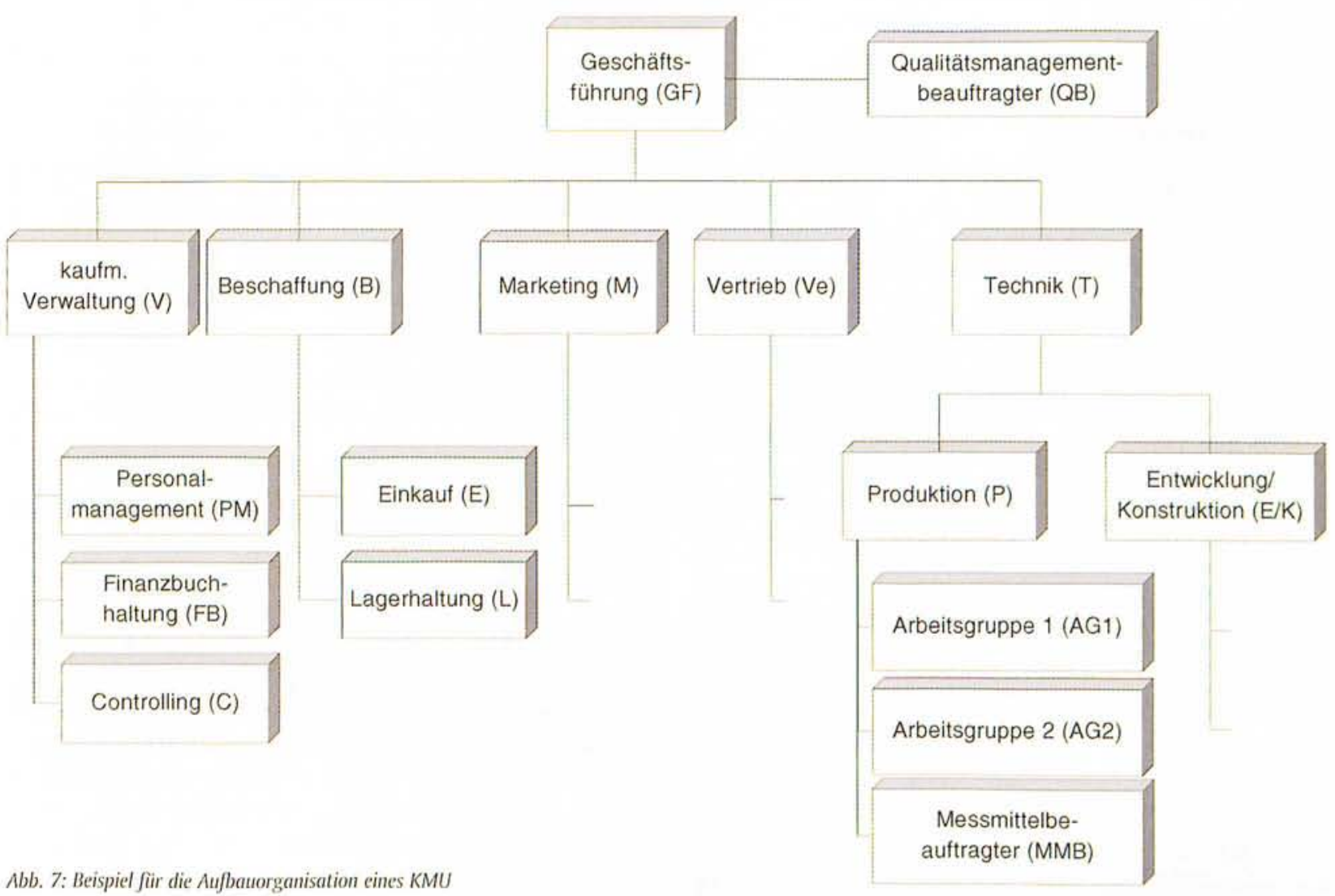




\section{geltende $(G) / z u$ \\ erarbeitende $(E)$ \\ Unterlagen}

G: Dokumente,

Qualitätsauf-

zeichnungen

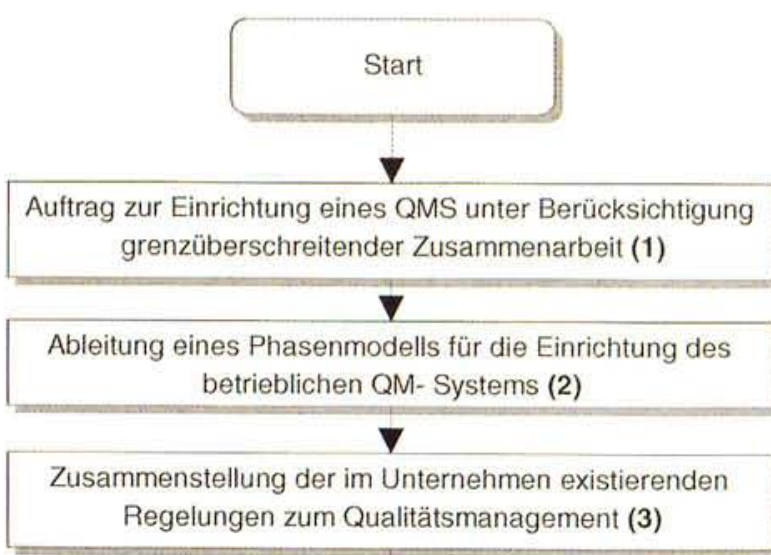

Verantwort-

lichkeiten

D: Durchführung

M: Mitarbeit

I: Information

D: GF

D: QB

D: QB

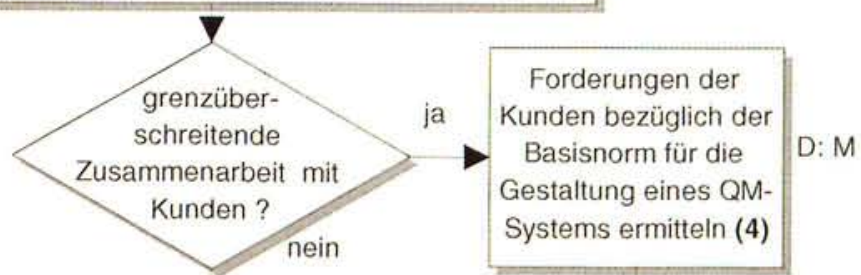

Kunden bezüglich der

Basisnorm für die

Gestaltung eines QM-

\section{: M}

E: Lieferantenübersicht

G: Liste der zugelassenen Lieferanten
G: Normen/Vorschriften

Erarbeitung einer Übersicht zu Basisnormen der bei den Lieferanten eingerichteten QMSystemen und der jeweiligen Zertifizierungsstelle (5)

\section{ja}
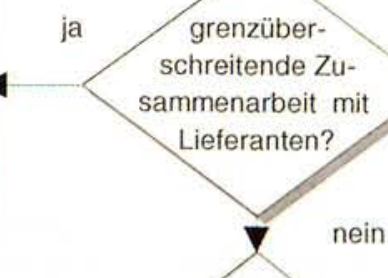

grenzüber-

schreitende Zu-

sammenarbeit mit Be-

hörden, Dienststellen u.a.? nein

Y u.a?

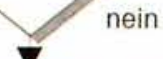

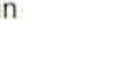

ja

Für die Zusamenarbeit mit Behörden,

Dienststellen u.a. im jeweiligen Partnerland spezielle Forderungen ermitteln (6)

D: B

E: Analyse-Auditcheckliste, Auditplan

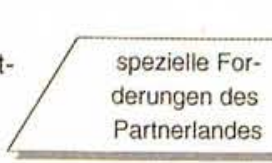

\section{$\nabla$}
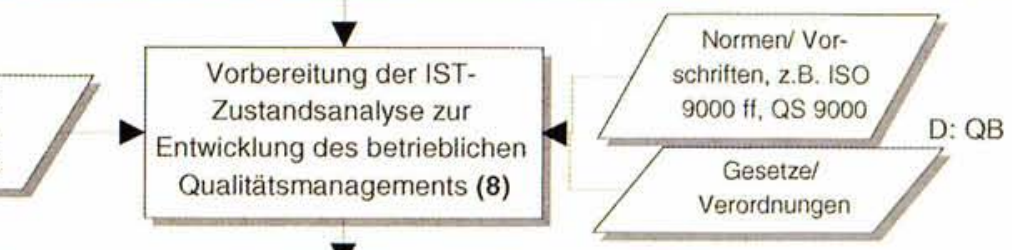

G: Analyse-Auditcheckliste

$$
\begin{gathered}
\text { Durchführung und Bewertung der IST-Zustandsanalyse } \\
\text { (Analyseaudit) (9) }
\end{gathered}
$$

D: QB

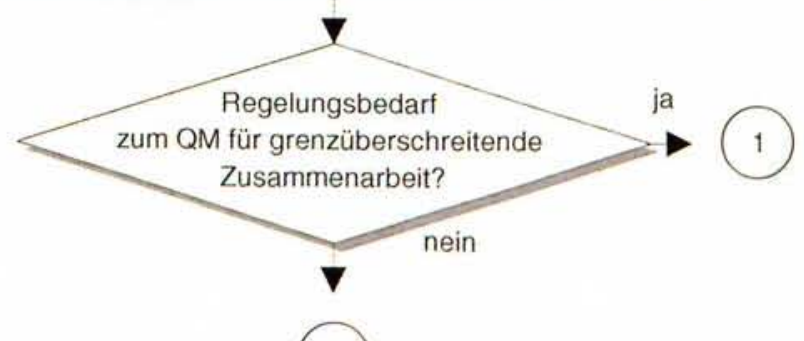


geltende (G)/ zu erarbeitende $(E)$

Unterlagen

E: Auditbericht

E: Ablaufplan

E: Ablaufplan, Dokumente,

Q-Aufzeichnungen

G: Dokumente,

Q-Aufzeichnungen

G: QM-Handbuch, Dokumente

G: QM-Handbuch, Dokumente
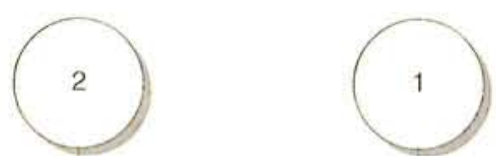

ngsbedarf fü grenzüberschreitende

Zusammenarbeit zusammenstellen (10)

Phasenmodell mit den grenzüberschreitenden Aufgaben und erforderlichen Aktivitäten untersetzten unter

Berücksichtigung der

Forderungen der

Normen-/Vorschriftenbasis (11)
Verantwortlichkeiten

D: Durchführung

M: Mitarbeit

I: Information

D: QB

M: GF, V, B, M, Ve, $T$

\section{$\mathrm{D}: \mathrm{QB}$}

M: GF, V, B, M, Ve, $T$

D: QB, GF, V, $B, M, V e, T$ erarbeiten und realisieren (12)

\section{$\nabla$}

Gestaltungslösungen, wenn möglich mit ausländischen Partnern erproben (13)

D: QB, GF, V, $B, M, V e, T$

Freigabe des QM- Systems (14)

D: GF

Arbeit mit dem implementierten QM- System (15)

D: QB, GF, V, $B, M, V e, T$
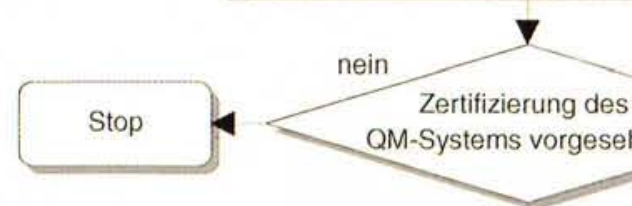

QM-Systems vorgesehen?

ja

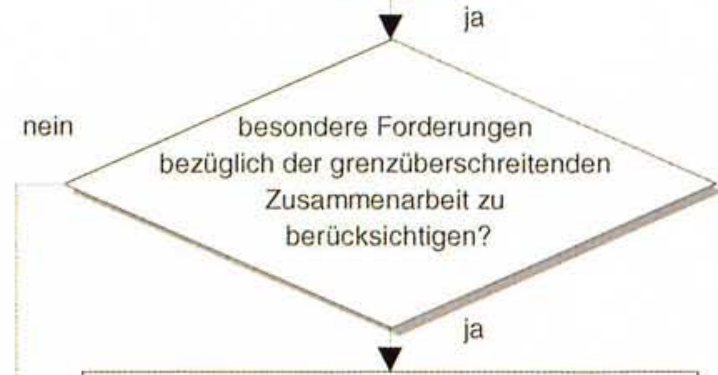

Forderungen aus grenzüberschreitender Zusammenarbeit zum Zwecke der Zertifizierung zusammenstellen (16)

D: QB

\section{$\nabla$}

Zertifizierungsstelle auswählen und beauftragen

(17)

D: GF

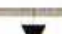

M: QB

Zertifizierungsverfahren realisieren (18)

\section{D: Zertifizie-} rungsstelle

G: Zetifikat

ausländische Partner über das Ergebnis des Zertifizierungsverfahren informieren (19)

D: $\mathrm{Ve}$

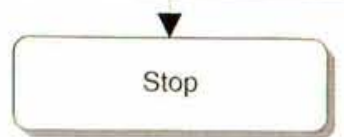




\section{Ablaufgestaltung der Einrichtung von $Q M$ - Systemen in KMU unter Berücksichtigung von Aspekten grenzüberschreitender Kooperation}

Prinzipiell kann die Einrichtung und Implementierung eines Qualitätsmanagementsystems in die Phasen Vorbereitung, Analyse, Realisierung und Erprobung gegliedert werden.

Grundlage für Zuständigkeitsregelungen bei der Gestaltung/Weiterentwicklung eines QM-Systems bildet die betriebliche Aufbauorganisation. Die in Abb. 7 dargestellte Aufbauorganisation orientiert sich am Stab-Linien-Prinzip.

Mit Hilfe von Abb. 8 werden Schritte bei der Implementierung eines QM-Systems in KMU unter Beriicksichtigung von Aspekten grenzüberschreitender Kooperation verdeutlicht. Inhaltliche Aufgabenstellungen der in Abb. 8 erfassten Aktivitäten enthält Tab. 4.

\section{Zusammenfassung}

Im vorliegenden Beitrag wurde von der Forderung ausgegangen, Aktivitäten der grenzüberschreitenden Kooperation in den QM-Systemen von KMU zu regeln. Dazu wurden Vorschläge für die methodische Vorgehensweise bei der Gestaltung und Weiterentwicklung betrieblicher QM-Systeme in KMU unterbreitet. Als besondere Schwerpunkte wurden die Identifikation des Handlungsbedarfes für die grenzüberschreitende Kooperation im Sinne einer angemessenen Erfüllung der Erwartungen und Forderungen interessierter Parteien eines Unternehmens, die in-/outputvernetzte Prozessgestaltung sowie Aufgabenfelder des Risikomanagements herausgestellt. Die Schrittfolge für die Gestaltung eines QM-Systems unter Berücksichtigung von Aspekten grenzüberschreitender Kooperation wird in einem Ablaufalgorithmus zusammengefasst.

\begin{tabular}{|c|c|}
\hline (1) & $\begin{array}{l}\text { e Einrichtung eines QMS unter Berücksichtigung grenzüberschreitender Zusammenarbeit kann durch die } \\
\text { gene Geschäftsührung („leitungsmotivierter Ansatz") oder/und durch Anforderungen der Interessenpartner wie } \\
\text { inden, Lieferanten oder ausländische Partner („interessenpartnermotivierter Ansatz") initiiert werden. }\end{array}$ \\
\hline (2) & $\begin{array}{l}\text { die Einrichtung des betrieblichen QM-Systems ist zunächst ein Konzept bezüglich Projektphasen und deren } \\
\text { iwerpunktaufgaben zu erarbeiten. }\end{array}$ \\
\hline (3) & $\begin{array}{l}\text { führte Dokumente und Qualitätsaufzeichnungen, z. B. Betriebsanweisungen, Organigramm, Prüfprotokolle. } \\
\text { ese sollten in die Einrichtung des QMS einbezogen werden. }\end{array}$ \\
\hline (4)-(7) & $\begin{array}{l}\text { der Vorbereitungsphase sind die Forderungen der ausländischen Partner (z. B. Kunden, Lieferanten, } \\
\text { ehörden) bezüglich der Basisnorm für die Gestaltung des QM-Systems zu ermitteln. In den vorliegenden } \\
\text { usführungen wird von der Nutzung der Norm ISO 9004:2000 ausgegangen. }\end{array}$ \\
\hline$(8)-(9)$ & $\begin{array}{l}\text { elstellungen der Ist-Zustandsanalyse sind: } \\
\text { - die Analyse und Beschreibung des Ist-Zustandes der Normenkonformität qualitätssichernder } \\
\text { Tätigkeiten im Unternehmen, } \\
\text { - die Ermittlung des Regelungsbedarfs, hier im Schwerpunkt für grenzüberschreitende } \\
\text { Zusammenarbeit, } \\
\text { die Schaffung von Grundlagen für die Erstellung eines unternehmensspezifischen Ablaufplanes für } \\
\text { die Einrichtung und Implementierung eines QM-Systems unter Berücksichtigung } \\
\text { grenzüberschreitender Anforderungen. }\end{array}$ \\
\hline & $\begin{array}{l}\text { Ist-Zustandsanalyse wird mit Hilfe einer unternehmensspezifischen Checkliste durchgeführt. In die Analyse- } \\
\text { iditcheckliste sind Anforderungen der Basisnorm, Anforderungen aus Gesetzen und Verordnungen, spezielle } \\
\text { forderungen des Partnerlandes, d.h. dort geltende Gesetze, Vorschriften u.s.w. zu integrieren. }\end{array}$ \\
\hline$(10)-(11)$ & $\begin{array}{l}\text { Is der Ist-Zustandsanalyse ergibt sich der unternehmensspezifische Regelungsbedarf zum QM für die } \\
\text { enzüberschreitende Zusammenarbeit. }\end{array}$ \\
\hline$(12)-(13)$ & $\begin{array}{l}\text { Nach Möglichkeit sollten die erarbeitenden Gestaltungslösungen mit ausländischen Partnern erprobt bzw. } \\
\text { mindestens abgestimmt werden. Dadurch werden mögliche Probleme bezüglich der Sprache, Terminologie, } \\
\text { Verhaltenskodizes, Unternehmensphilosophie aufgezeigt. Ziel dieser Erprobung ist weiterhin die Prüfung der } \\
\text { Kompatibiliät der Gestaltungslösungen des betrieblichen Qualitätsmanagementsystems mit den Regelungen } \\
\text { ausländischer Partner. }\end{array}$ \\
\hline$(14)$ & ausländischen Partnern kann das QM-System freigegeben werden. \\
\hline (15) & $\begin{array}{l}\text { Implementierung des QM-Systems ist darauf zu achten, dass die ausländischen Partner nach den } \\
\text { rtraglich vereinbarten Vorschriften und Festlegungen arbeiten. Des weiteren spielt die Bewertung der } \\
\text { plementierten QM-Systeme durch Qualitätsaudits und Qualitätsreviews eine besondere Rolle. So sollten die } \\
\text { Idits wenn möglich nach gleichen Regelungen und Checklisten bei den Partnern durchgeführt werden. }\end{array}$ \\
\hline$(16-(18)$ & 5 \\
\hline
\end{tabular}

Tab. 4: Inhaltliche Schwerpunktaufgaben bei der Integration von Aspekten der grenzüberschreitenden Kooperation in QM-Systemen von KMU 


\section{Literatur}

(1) N.N., Das Auslandsgeschäft - Motor unserer Volkswirtschaft, in: Erfolg im Ausland, weltweit aktiv; Ratgeber für kleine und mittlere Unternehmen, Bundesministerium für Wirtschaft, 3. Auflage, Juli 2000

[2] Autorenteam, Qualitätsmanagement und grenzüberschreitende Kooperation, Leitfaden zur Einbeziehung von Aspekten der grenzuiberschreitenden Kooperation bei der Gestaltung von Qualitätsmanagementsystemen in kleinen und mittleren Unternehmen; Pilotprojekt im Auftrag der Handwerkskammer Frankfurt (Oder)

[3] N.N., Umweltmanagement im Spannungsfeld zwischen Ökologie und Ökonomie, Organisationsforum Wirtschaftskongress e. $\mathrm{V}$.

\section{Autoren}

\section{Dipl.-Betriebswirtin (FH) Nicole Petschick}

Technische Fachhochschule Wildau

Fachbereich Ingenieurwesen/Wirtschaftsingenieurwesen

Tel. (0 33 75) 508-915

\section{Prof. Dr.-Ing. habil. Gerd Stegemann}

Technische Fachhochschule Wildau

Fachbereich Ingenieurwesen/Wirtschaftsingenieurwesen

Tel. (0 33 75) 508-914

E-Mail: qmstegem@igw.tfh-wildau.de 